\title{
Nontrivial topological phase with a zero Chern number
}

\author{
H. C. Wu, L. Jin $\odot,^{*}$ and Z. Song $\odot^{\dagger}$ \\ School of Physics, Nankai University, Tianjin 300071, China
}

(Received 4 January 2020; accepted 10 July 2020; published 22 July 2020)

\begin{abstract}
A two-dimensional (2D) topological band is characterized by the (first) Chern number. The zero and nonzero Chern numbers usually represent the trivial and nontrivial band topologies, respectively. In this paper, we study an extended Qi-Wu-Zhang model that hosts the topological band with a zero Chern number. We show that the zero Chern number band is topologically nontrivial, characterized by the half-integer wave polarization. The nontrivial topology is manifested by the anisotropic in-gap edge states, which are verified to be robust against 2D particle-hole symmetric disorders.
\end{abstract}

DOI: 10.1103/PhysRevB.102.035145

\section{INTRODUCTION}

Topological insulators and superconductors have many potential applications because of their topologically nontrivial bands [1,2]. They support topological transport at the edges and interfaces, and the transport is robust against the presented imperfections [3]. Topological insulators in one, two, three, and even higher dimensions with a synthetic dimension are proposed and experimentally investigated in cold atomic gases, photonic crystals, and acoustic lattices [4-27].

Two-dimensional (2D) topological systems have been intensively investigated in topological physics [28-39]. The Chern insulator has successfully explained the 2D quantum Hall effect under a magnetic field [40-42] and the quantum anomalous Hall effect [43-48]. A prototypical Chern insulator is the Qi-Wu-Zhang (QWZ) model [49]. The (first) Chern number associated with the energy band is a topological invariant, which is a quantized Berry flux because the integration of Berry curvature over the whole Brillouin zone (BZ) indicates the band topology of 2D topological phases [50].

The nonzero Chern number yields the topologically nontrivial phase; the difference between the Chern numbers of bands indicates the number of topologically protected edge states in the band gap [51,52]. For example, in the 2D quantum Hall phase formed through breaking the time-reversal symmetry of the 2D system with a pair of Dirac cones, the band gap and a pair of edge states connecting the upper and lower bands are created; in contrast, breaking the inversion symmetry of the 2D system with a pair of Dirac cones creates the topologically trivial band that is characterized by the zero

\footnotetext{
*jinliang@nankai.edu.cn

† songtc@nankai.edu.cn
}

Published by the American Physical Society under the terms of the Creative Commons Attribution 4.0 International license. Further distribution of this work must maintain attribution to the author(s) and the published article's title, journal citation, and DOI.
Chern number, and the topologically protected edge states are absent [53]. Recently, in an isotropic 2D topological insulator with the zero integral of the Berry curvature over the BZ, the zero Berry curvature in the whole BZ except at the $C_{4 v}$ invariant lines of band degeneracy was reported [54]; the Chern number associated with the energy band vanishes as well. However, the 2D Zak phase [55], the Berry connection integrated over the whole $\mathrm{BZ}$ as the generalization of the onedimensional (1D) Zak phase, is available for the topological characterization.

In this paper, we study an anisotropic 2D square lattice with effective spin-orbital interaction alternatively introduced along one direction. The 2D lattice is an array of coupled Creutz ladders and can be taken as an extension of the QWZ model. The topological properties of different phases of the anisotropic 2D lattice are investigated. Beyond the traditional understanding that the topological phase with a nonzero Chern number supports a pair of chiral edge states, we find that the anisotropic 2D lattice also has a topologically nontrivial phase with a zero Chern number but nonzero Berry curvature. A pair of topologically protected in-gap edge states is present in the topological phase with a zero Chern number. The ingap edge states are robust to the $2 \mathrm{D}$ particle-hole symmetric disorders; this verifies the topological protection. The 2D Zak phase is employed for the topological characterization and corresponds to the fractional wave polarization. In our work, the validity of the $2 \mathrm{D}$ Zak phase is ensured by the fact that the singularity point is absent in the whole BZ under the properly chosen gauge for the topological phase with a zero Chern number. Our findings deepen the understanding of the topologically nontrivial phase present in other topological systems.

The remainder of this paper is organized as follows. In Sec. II, the anisotropic 2D lattice is introduced. The energy bands and phase diagram are shown. In Sec. III, the topological characterization of the Chern number and the 2D Zak phase are discussed, particularly the topologically nontrivial phase with a zero Chern number. In Sec. IV, the analytical solution of edge states is given. In Sec. V, the robustness of the topologically protected in-gap edge states is verified. In Sec. VI, the conclusion is drawn. 

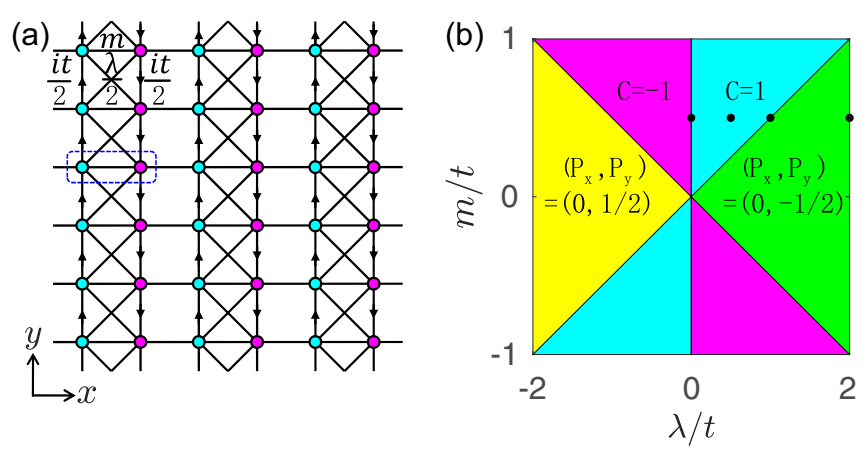

FIG. 1. (a) Schematic of the 2D square lattice. The Peierls phase factor $e^{i \pi / 2}$ in the nonreciprocal coupling it $/ 2$ in the vertical direction induces $\pm \pi$ magnetic flux in each plaquette. (b) Phase diagram in the $\lambda-m$ parameter space; $t$ is taken as the unit. The Chern numbers are nonzero in the magenta and cyan regions. The Chern number is $C=0$ in the yellow and green regions with the polarizations given; the half-integer polarization in the $y$ direction indicates the nontrivial topology. The black dots indicate the parameters chosen in Fig. 2.

\section{ANISOTROPIC 2D SQUARE LATTICE}

We consider an anisotropic 2D square lattice; the schematic is given in Fig. 1(a). The coupling strengths in the horizontal and vertical directions are uniform, being $m$ and $t / 2$, respectively. The Peierls phase factors presented in the vertical couplings induce the $\pm \pi$ effective magnetic flux in each plaquette. The lattice is divided into two sublattices colored in cyan and magenta, respectively. The cross coupling is an effective spin-orbital coupling; it alternatively presents in the horizontal direction, and the coupling strength is $\lambda / 2$.

The anisotropic 2D lattice is a two-band system; the unit cell is marked by the blue dashed rectangle. The Bloch Hamiltonian obtained after Fourier transformation is

$$
H(\mathbf{k})=B_{x} \sigma_{x}+B_{y} \sigma_{y}+B_{z} \sigma_{z}
$$

where the effective magnetic field is

$$
\begin{aligned}
& B_{x}=m+m \cos k_{x}+\lambda \cos k_{y}, \\
& B_{y}=m \sin k_{x}, \quad B_{z}=t \sin k_{y},
\end{aligned}
$$

and $\sigma_{x, y, z}$ is the Pauli matrix for spin 1/2. The Bloch Hamiltonian $H(\mathbf{k})$ can be taken as an extended QWZ model in the sense that $H(\mathbf{k})$ under the unitary operation

$$
\hat{R}=\frac{1}{\sqrt{2}}\left(\begin{array}{cc}
1 & 1 \\
i & -i
\end{array}\right)
$$

yields

$$
\hat{R} H(\mathbf{k}) \hat{R}^{-1}=B_{y} \sigma_{x}+B_{z} \sigma_{y}+B_{x} \sigma_{z},
$$
the effective magnetic field being $\left(B_{y}, B_{z}, B_{x}\right)=$ $\left(m \sin k_{x}, t \sin k_{y}, m+m \cos k_{x}+\lambda \cos k_{y}\right)$. The rotated Bloch Hamiltonian $\hat{R} H(\mathbf{k}) \hat{R}^{-1}$ at $m=\lambda=t$ reduces to the $\mathrm{QWZ}$ model in a nontrivial gapped phase with the Chern number $C=1$ [52]. We have $\hat{R}^{3}=e^{i \pi / 4} \sigma_{0}$ and $\hat{R}^{3} H(\mathbf{k}) \hat{R}^{-3}=H(\mathbf{k})$, where $\sigma_{0}$ is the $2 \times 2$ identity matrix.

The Bloch Hamiltonian $H(\mathbf{k})$ has particle-hole symmetry $\hat{C} H(\mathbf{k}) \hat{C}^{-1}=-H(-\mathbf{k})$, with $\hat{C}=\sigma_{z} K$, where $K$ is the complex conjugation and the inversion symmetry $\hat{P} H(\mathbf{k}) \hat{P}^{-1}=$ $H(-\mathbf{k})$, with $\hat{P}=\sigma_{x}$. The Bloch Hamiltonian $H(\mathbf{k})$ possesses the same symmetries as the QWZ model, and both of them
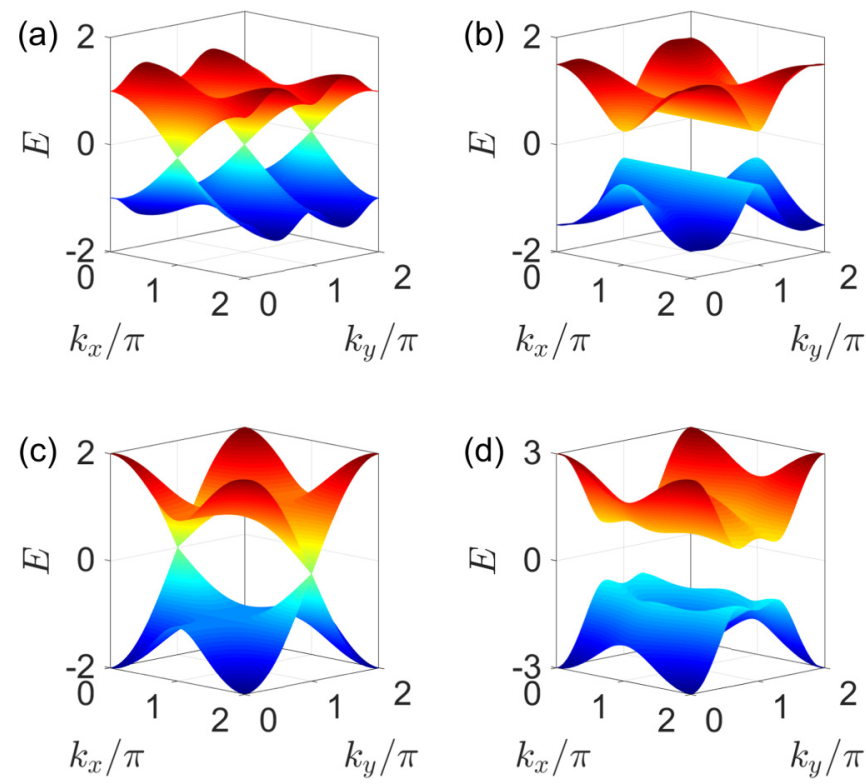

FIG. 2. Energy bands of the anisotropic 2D square lattice. (a) $\lambda=0$, (b) $\lambda=1 / 2$, (c) $\lambda=1$, (d) and $\lambda=2$; other parameters are $t=1$ and $m=1 / 2$, as indicated by the black dots in Fig. 1(b).

belong to class $\mathrm{D}$ in the tenfold way of Altland-Zirnbauer classification [56-58]. However, we demonstrate the existence of in-gap topological edge states in the anisotropic 2D lattice [Fig. 1(a)] in the zero Chern number phase in contrast to the nonexistence of edge states in the zero Chern number phase of the QWZ model.

The spectrum of $H(\mathbf{k})$ is symmetric about zero energy; the band energy is given by

$$
E\left(k_{x}, k_{y}\right)= \pm B= \pm \sqrt{B_{x}^{2}+B_{y}^{2}+B_{z}^{2}}
$$

Along the vertical direction of the 2D lattice, the unit cells form the Creutz ladder [24,59]. The coupling strength $t$ does not affect the band gap closing or the topological phase of the Creutz ladder. Thus, we can take the vertical coupling strength $t$ as unity without loss of generality. From the expression of the band energy $E\left(k_{x}, k_{y}\right)$ after substituting $B_{x, y, z}$ as functions of the system parameters, we find that the band gap closes under the condition $|m / \lambda|=1 / 2$ or $\lambda=0$. The band degeneracy point locates at $\left(k_{x}, k_{y}\right)=(0, \pi)$ for $m / \lambda=1 / 2$ and locates at $\left(k_{x}, k_{y}\right)=(0,0)$ for $m / \lambda=-1 / 2$. For $\lambda=0$ and $m \neq 0$, the band gap closes at $\left(k_{x}, k_{y}\right)=(\pi, 0)$ and $\left(k_{x}, k_{y}\right)=(\pi, \pi)$. Otherwise, the two energy bands are gapped as the insulator phase.

The phase diagram of the Bloch Hamiltonian $H(\mathbf{k})$ is shown in Fig. 1(b), where the band gap closes at the black solid lines and the system is gapped in the colored regions, with topological numbers marked in the phase diagram. The energy bands of the Bloch Hamiltonian $H(\mathbf{k})$ are depicted in Fig. 2 at several fixed coupling strengths. Figure 2(a) is a gapless phase with vanishing cross coupling $\lambda / 2$. In the presence of cross coupling $\lambda / 2$, the band gap appears for cross coupling $\lambda=1 / 2$ in Fig. 2(b), closes for cross coupling $\lambda=1$ in Fig. 2(c), and reopens at a larger cross coupling, $\lambda=2$, in 
Fig. 2(d). The band-touching points are fixed at $k_{x}=\pi$ for $\lambda=0$ and at $k_{x}=0$ for $\lambda= \pm 2 m$, as mentioned above.

\section{TOPOLOGICAL PHASES}

Topological properties of $H(\mathbf{k})$ are encoded in the wave functions of the energy bands. The wave functions of the upper band (with subscript + ) and the lower band (with subscript -) are given by

$$
\left|\psi_{ \pm}(\mathbf{k})\right\rangle=\frac{1}{N_{ \pm}}\left(\begin{array}{c}
B_{z} \pm B \\
B_{x}+i B_{y}
\end{array}\right),
$$

where $N_{ \pm}=\left[2 B\left(B \pm B_{z}\right)\right]^{1 / 2}$ is the normalization coefficient and $\left\langle\psi_{ \pm}(\mathbf{k}) \mid \psi_{ \pm}(\mathbf{k})\right\rangle=1$.

In the gapped phase, the Chern number is a topological invariant that characterizes the topological properties of the anisotropic 2D lattice. The Chern number of the energy band is well defined as the integral of Berry curvature over the entire BZ,

$$
C_{ \pm}=\frac{1}{2 \pi} \iint_{\mathrm{BZ}} d k_{x} d k_{y} \Omega_{ \pm}
$$

where the Berry curvature is defined as $\Omega_{ \pm}=\nabla \times A_{ \pm}$and the Berry connection is defined as $A_{ \pm}=-i\left\langle\psi_{ \pm}(\mathbf{k})|\nabla| \psi_{ \pm}(\mathbf{k})\right\rangle$. The upper and lower bands are indicated by the subscripts + and -, respectively [60]. The Chern numbers of the two bands satisfy $C_{+}+C_{-}=0$; we denote $C=C_{-}$for convenience. The phase diagram depicted in Fig. 1(b) shows the Chern numbers in the parameter space of the Bloch Hamiltonian $H(\mathbf{k})$.

The number and position of singularities in the BZ depend on the gauge chosen. The number of irremovable singularities determines the Chern number. In the region $m / \lambda>1 / 2$ $(m / \lambda<-1 / 2)$, there exists at least one singularity in the BZ that cannot be removed under any chosen gauge. The singularity in the BZ is the point where the Berry connection $A_{ \pm}$is not well defined; for the upper band $\left|\psi_{+}(\mathbf{k})\right\rangle$, it appears at certain $\mathbf{k}$ where $B_{x}=B_{y}=0$ and $B=-B_{z}$, and for the lower band $\left|\psi_{-}(\mathbf{k})\right\rangle$, it appears at certain $\mathbf{k}$ where $B_{x}=B_{y}=0$ and $B=B_{z}$. The Chern number calculated in this situation is $C=1(C=-1)$; the nonzero Chern number indicates the nontrivial band topology; consequently, a pair of chiral edge states localized at the boundary of the system under the open boundary condition (OBC) is expected. In the regions $|m / \lambda|<1 / 2$, the Chern number is $C=0$. We emphasize that the Berry curvature is continuous in the whole $\mathrm{BZ}$ and nonvanishing, except the Berry curvature crosses zero from a positive value to a negative value or vice versa. The Berry curvature in the zero Chern number phase has different features than that in the 2D square lattice in Ref. [54]. Notably, $\hat{R}^{2} H(\mathbf{k}) \hat{R}^{-2}$ under twice the rotation operations characterize $H(\mathbf{k})$ under a different gauge. The wave functions change into $\left|\psi_{ \pm}^{\prime \prime}(\mathbf{k})\right\rangle=N_{ \pm}^{\prime \prime-1}\left(B_{y} \pm B, B_{z}+i B_{x}\right)^{T}$, with the normalization coefficient $N_{ \pm}^{\prime \prime}=\left[2 B\left(B \pm B_{y}\right)\right]^{1 / 2}$. In this situation, $B_{x}$ and $B_{z}$ cannot vanish simultaneously in the BZ. Consequently, the Berry connection $A_{ \pm}^{\prime \prime}=-i\left\langle\psi_{ \pm}^{\prime \prime}(\mathbf{k})|\nabla| \psi_{ \pm}^{\prime \prime}(\mathbf{k})\right\rangle$ has no singularity, and two bands have a zero Chern number. In most cases, the zero Chern number indicates the trivial band topology [52]; however, the band topology with the zero Chern number in the anisotropic 2D lattice model is nontrivial. We will show that the system under the $\mathrm{OBC}$ in the $y$ direction has a pair of in-gap edge states which is topologically protected and robust to disorders.

To elucidate the nontrivial topology and the presence of topological in-gap edge states in the region with a zero Chern number, we employ another topological characterization: The wave polarization, which is related to the integral of the Berry connection in the whole $\mathrm{BZ}$ and is equal to the $2 \mathrm{D} \mathrm{Zak}$ phase divided by $2 \pi$ [54]. The fractional wave polarization in a certain direction of the 2D lattice predicts the nontrivial topology. The projections of the wave polarization in the $x$ and $y$ directions are given by

$$
\begin{aligned}
P_{ \pm, x} & =\frac{1}{(2 \pi)^{2}} \iint_{\mathrm{BZ}} A_{ \pm, x}\left(k_{x}, k_{y}\right) d k_{x} d k_{y}, \\
P_{ \pm, y} & =\frac{1}{(2 \pi)^{2}} \iint_{\mathrm{BZ}} A_{ \pm, y}\left(k_{x}, k_{y}\right) d k_{x} d k_{y},
\end{aligned}
$$

where $\quad A_{ \pm, x}\left(k_{x}, k_{y}\right)=-i\left\langle\psi_{ \pm}\left(k_{x}, k_{y}\right)\left|\partial_{x}\right| \psi_{ \pm}\left(k_{x}, k_{y}\right)\right\rangle \quad$ and $A_{ \pm, y}\left(k_{x}, k_{y}\right)=-i\left\langle\psi_{ \pm}\left(k_{x}, k_{y}\right)\left|\partial_{y}\right| \psi_{ \pm}\left(k_{x}, k_{y}\right)\right\rangle$ are the Berry connections in the $x$ and $y$ directions, respectively. The polarizations in the $x$ and $y$ directions are given in the phase diagram of Fig. 1(b). The nonzero half-integer polarization predicts the presence of in-gap edge states for the phase with a zero Chern number, in which the number of singularities in the whole BZ is zero under the properly chosen gauge. The polarization calculated from the Berry connection under the proper gauge is capable of correctly predicting the topological in-gap edge states; the one-half projected polarization in the $y$ direction in the $C=0$ phase indicates the presence of topological in-gap edge states in the $y$ direction.

The energy bands of the 2D lattice under the $\mathrm{OBC}$ in the $x$ or $y$ direction are depicted in Fig. 3 for two different topological phases with Chern numbers $C=0$ and $C=1$. For the topologically nontrivial phase with a nonzero Chern number $C=1$, Fig. 3(a) [Fig. 3(c)] plots the energy bands under the periodic boundary condition (PBC) in the $y(x)$ direction but under the OBC in the $x(y)$ direction. In Figs. 3(a) and 3(c), we observe a pair of chiral edge states; they are topologically protected and localized on the two open boundaries. In Fig. 3(a), the blue edge state localizes at the left boundary with velocity $v_{y}<0$ moving downward, and the green edge state localizes at the right boundary with $v_{y}>0$ moving upward; in Fig. 3(c), the blue edge state localizes at the bottom boundary with velocity $v_{x}>0$ moving rightward, and the green edge state localizes at the top boundary with $v_{x}<0$ moving leftward. Thus, the edge states have a definite chirality, moving counterclockwise in the 2D lattice. For the topological phase with a zero Chern number, Fig. 3(b) plots the energy bands under the PBC in the $y$ direction but under the OBC in the $x$ direction, the polarization in the $x$ direction is $P_{-, x}=0$; consequently, topologically protected edge states are absent. Figure 3(d) plots the energy band under the PBC in the $x$ direction but under the $\mathrm{OBC}$ in the $y$ direction. In this phase, the topological protection of the in-gap edge states is guaranteed by the one-half polarization in the $y$ direction $P_{-, y}=-1 / 2$. A pair of in-gap edge states exists; they are localized on the top and bottom boundaries in the $y$ direction.

The quantized 2D Zak phase in the $C=0$ phase can be understood as follows. The 1D Zak phase for a fixed $k_{x}$ is defined as $Z_{y, \pm}\left(k_{x}\right)=\int_{-\pi}^{\pi} A_{y, \pm}\left(k_{x}, k_{y}\right) d k_{y}$. To be specific, 

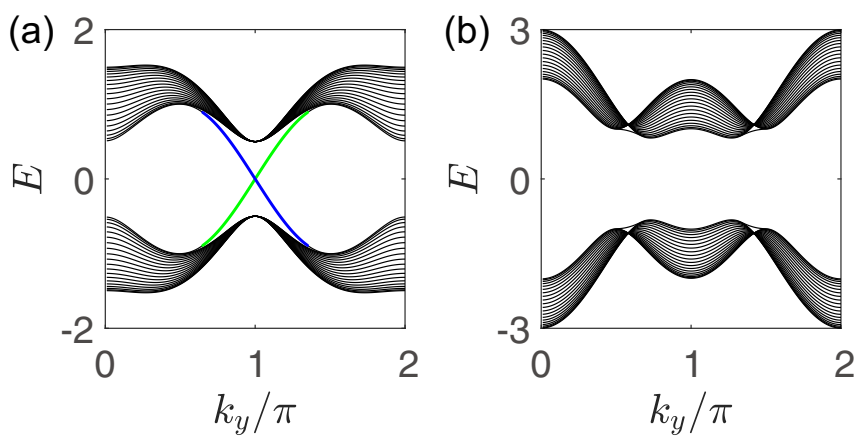

(c)
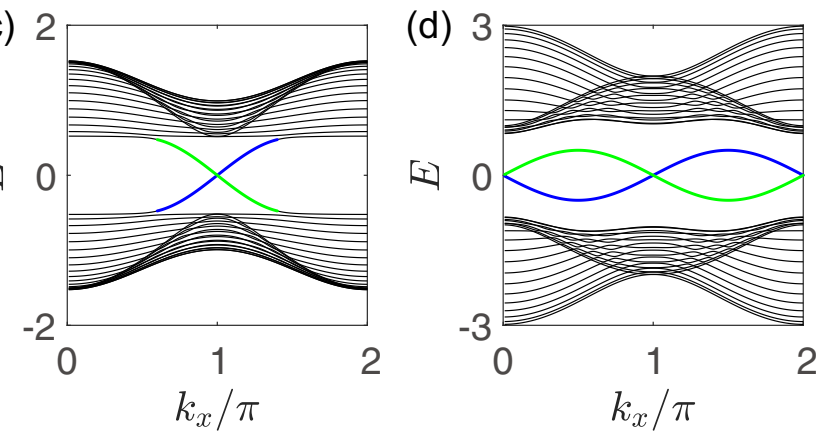

FIG. 3. Energy spectra of the 2D square lattice under different OBCs. (a) and (b) plot the energy spectra under the PBC in the $y$ direction but under the $\mathrm{OBC}$ in the $x$ direction for different phases. (c) and (d) plot the energy spectra under the PBC in the $x$ direction but under the $\mathrm{OBC}$ in the $y$ direction for different phases. The parameters are $t=1, m=1 / 2$, and $\lambda=1 / 2$ in (a) and (c) for $C=1$ and $\lambda=2$ in (b) and (d) for $C=0$.

we have $Z_{y, \pm}\left(k_{x}\right)=\int_{-\pi}^{\pi}\left(B_{z} \partial_{\mathrm{y}} B_{x}-B_{x} \partial_{\mathrm{y}} B_{z}\right) /\left[2 B\left(B \pm B_{y}\right)\right] d k_{y}$ for the wave function $\left|\psi_{ \pm}^{\prime \prime}(\mathbf{k})\right\rangle$ of the rotated Hamiltonian $\hat{R}^{2} H(\mathbf{k}) \hat{R}^{-2}$ in the topological characterization. From $B_{x}\left(k_{x}\right)=B_{x}\left(-k_{x}\right), B_{y}\left(k_{x}\right)=-B_{y}\left(-k_{x}\right), \quad$ and $\quad B_{z}\left(k_{x}\right)=$ $B_{z}\left(-k_{x}\right)$, we have the relation $Z_{y,+}\left(-k_{x}\right)=Z_{y,-}\left(k_{x}\right)$; consequently, we obtain $Z_{y,+}\left(-k_{x}\right)+Z_{y,+}\left(k_{x}\right)=Z_{y,-}\left(k_{x}\right)+$ $Z_{y,+}\left(k_{x}\right)=\int_{-\pi}^{\pi}\left(B_{z} \partial_{\mathrm{y}} B_{x}-B_{x} \partial_{\mathrm{y}} B_{z}\right) /\left(B_{x}^{2}+B_{z}^{2}\right) d k_{y}$, which is an integer of $2 \pi$. After straightforward calculation, we have $Z_{y,+}\left(k_{x}\right)+Z_{y,+}\left(-k_{x}\right)=-2 \pi$ for $\lambda>0$. Following the same approach, we have $Z_{x, \pm}\left(k_{y}\right)=\int_{-\pi}^{\pi} A_{x, \pm}\left(k_{x}, k_{y}\right) d k_{x}=$ $\int_{-\pi}^{\pi}\left(B_{z} \partial_{\mathrm{x}} B_{x}-B_{x} \partial_{\mathrm{x}} B_{z}\right) /\left[2 B\left(B \pm B_{y}\right)\right] d k_{x}$; thus, we obtain $Z_{x,+}\left(-k_{y}\right)+Z_{x,+}\left(k_{y}\right)=0$ from the fact that $B_{x}\left(k_{y}\right)=$ $B_{x}\left(-k_{y}\right), B_{y}\left(k_{y}\right)=B_{y}\left(-k_{y}\right)$, and $B_{z}\left(k_{y}\right)=-B_{z}\left(-k_{y}\right)$. These results reproduce the quantized $1 \mathrm{D}$ Zak phase at both $k_{x}=0, \pi$ and $k_{y}=0, \pi$ as a consequence of the inversion symmetry [61], which is $-\pi$ and zero for $\lambda>0$, respectively. Therefore, the polarization integrated in the whole BZ is a half-integer, and the $2 \mathrm{D}$ Zak phase is quantized, being an integer of $\pi$.

A key point for the in-gap edge states is that they are present in the entire zero to $2 \pi$ region of the momentum $k_{x}$. The continuity of the in-gap feature of the edge state is related to the absence of singularity, where the $2 \mathrm{D} \mathrm{Zak}$ phase is well defined and is suitable for the topological characterization of the $C=0$ phase. The ratio between $m$ and $\lambda$ is critical for the presence of the in-gap edge states. This is explained through the comparison between the anisotropic 2D lattice and the QWZ model. The Bloch Hamiltonian $H(\mathbf{k})$ of the anisotropic 2D lattice differs from the QWZ model in the sense that the couplings in the horizontal direction, the couplings in the vertical direction, and the cross couplings are all independent of each other, while the couplings in the QWZ model are uniform, except the horizontal couplings are staggered. In the QWZ model, the effective magnetic field is $B_{x}=m+t \cos k_{x}+t \cos k_{y}, B_{y}=t \sin k_{x}$, and $B_{z}=t \sin k_{y}$. The gapped phase with vanishing Chern number $|m / t|>2$ is topologically trivial without any edge states in either the vertical direction or the horizontal direction. After calculating the wave polarization in the QWZ model, we obtain zeros in both directions, $\left(P_{ \pm, x}, P_{ \pm, y}\right)_{\mathrm{QWZ}}=(0,0)$. The vanishing wave polarizations are in accord with the nonexistence of edge states in the topologically trivial phase of the QWZ model.

\section{EDGE STATES}

In this section, the wave functions of the topological edge states in the topologically nontrivial phases are presented. We consider that $N=2 N_{x} \times N_{y}$ is the total site number for the anisotropic 2D lattice; $2 N_{x}$ is the number of columns, and $N_{y}$ is the number of rows. The topological edge states are obtained from the Schrödinger equations for the 1D projection lattices.

For the system under the OBC in the $x$ direction, the 1D projection lattice is a dimerized chain with staggered couplings $m+\lambda \cos \left(k_{y}\right)$ and $m$, and the on-site potentials $t \sin \left(k_{y}\right)$ and $-t \sin \left(k_{y}\right)$ are alternatively presented. The site number of the 1D projection lattice chain is $2 N_{x}$. The left edge state $\left|\psi_{L}\right\rangle$ localizes at the left boundary of the chain; the corresponding Schrödinger equations read

$$
\begin{aligned}
E_{L} \psi_{2 j-1} & =m \psi_{2 j-2}+t \sin k_{y} \psi_{2 j-1}+\left(m+\lambda \cos k_{y}\right) \psi_{2 j}, \\
E_{L} \psi_{2 j} & =\left(m+\lambda \cos k_{y}\right) \psi_{2 j-1}-t \sin k_{y} \psi_{2 j}+m \psi_{2 j+1},
\end{aligned}
$$

where $j=1,2,3, \ldots, N_{x}$ is the index in the $x$ direction and $\psi_{0}=\psi_{2 N_{x}+1}=0$. The left edge state $\left|\psi_{L}\right\rangle$ is staggered and decays from the left boundary of the lattice, and we have $\psi_{2 j}=0$ from the recurrence equations for $N_{x} \gg 1$. From the Schrödinger equation, we obtain the eigenenergy $E_{L}\left(k_{y}\right)=$ $t \sin k_{y}$ and take $\psi_{1}=1$ to express the profile of the edge states instead of normalizing the edge states without loss of generality. The wave function of the left edge state reads

$$
\psi_{2 j-1}=\left[1+(\lambda / m) \cos k_{y}\right]^{j-1}, \quad \psi_{2 j}=0 .
$$

The right edge state $\left|\psi_{R}\right\rangle$ localizes at the right boundary of the chain, and the corresponding Schrödinger equations read

$$
\begin{aligned}
E_{R} \psi_{2 j} & =m \psi_{2 j+1}-t \sin k_{y} \psi_{2 j}+\left(m+\lambda \cos k_{y}\right) \psi_{2 j-1}, \\
E_{R} \psi_{2 j-1} & =\left(m+\lambda \cos k_{y}\right) \psi_{2 j}+t \sin k_{y} \psi_{2 j-1}+m \psi_{2 j-2},
\end{aligned}
$$

where $j=1,2,3, \ldots, N_{x}$ is the index in the $x$ direction and $\psi_{0}=\psi_{2 N_{x}+1}=0$. The right edge state $\left|\psi_{R}\right\rangle$ is staggered and decays from the right boundary of the chain; similarly, we have $\psi_{2 j-1}=0$, and the eigenenergy of the right edge state is obtained as $E_{R}\left(k_{y}\right)=-t \sin k_{y}$. If we take $\psi_{2 N_{x}}=1$, the wave function of the right edge state is given by

$$
\psi_{2 N_{x}+2-2 j}=\left[1+(\lambda / m) \cos k_{y}\right]^{j-1}, \quad \psi_{2 N_{x}+1-2 j}=0 .
$$



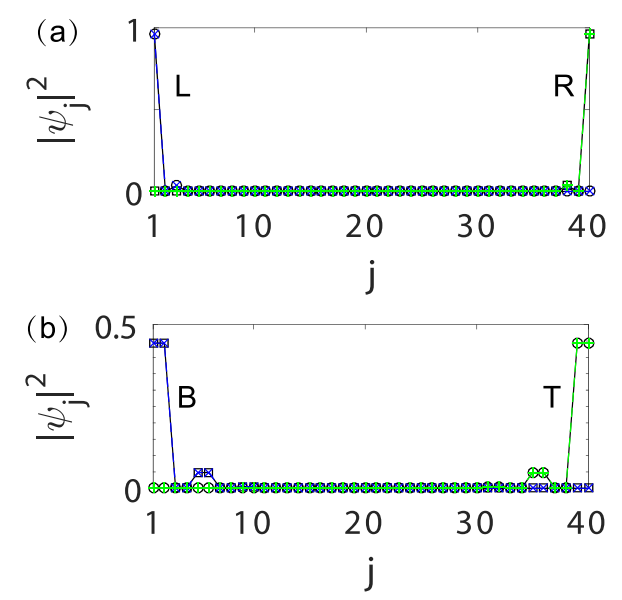

FIG. 4. Edge states for the 1D (quasi-1D) projection under the OBC. (a) $\lambda=1 / 2, k_{y}=4 \pi / 5$; (b) $\lambda=2, k_{x}=4 \pi / 5$. The black circles and squares are the numerical simulations, and the crosses are the analytical results. The other parameters are $t=1, m=1 / 2$.

For the 2D lattice under the $\mathrm{OBC}$ in the $y$ direction, topological edge states are always present. The projection of the 2D lattice in the $y$ direction is a quasi-1D ladder with two legs; the total site number is $2 N_{y}$. The topological edge states in the $C=1$ phase and the in-gap edge states in the $C=0$ phase have identical expressions of energy and the wave function. The topological edge states are obtained following the same approach as discussed previously. The bottom edge state $\left|\psi_{B}\right\rangle$ localizes at the bottom boundary of the ladder and has eigenenergy $E_{B}\left(k_{x}\right)=-m \sin k_{x}$. If we take $\psi_{1}=1$, the wave function of the bottom edge state can be expressed as

$$
\begin{aligned}
\psi_{3} & =\frac{2\left(i E_{B}-m-m e^{-i k_{x}}\right)}{t+\lambda} \psi_{1}, \\
\psi_{2 j+3} & =\frac{2\left(i E_{B}-m-m e^{-i k_{x}}\right)}{t+\lambda} \psi_{2 j+1}+\frac{t-\lambda}{t+\lambda} \psi_{2 j-1}, \\
\psi_{2 j} & =-i \psi_{2 j-1},
\end{aligned}
$$

where $j=1,2,3, \ldots, N_{y}-2$ is the index in the $y$ direction; $\psi_{2 N_{y}-2}=-i \psi_{2 N_{y}-3}$, and $\psi_{2 N_{y}}=-i \psi_{2 N_{y}-1}$. The top edge state $\left|\psi_{T}\right\rangle$ localizes at the top boundary of the ladder and has eigenenergy $E_{T}\left(k_{x}\right)=m \sin k_{x}$. If we take $\psi_{2 N_{y}}=1$, the wave function of the top edge state is given by

$$
\begin{aligned}
\psi_{2 N_{y}-2} & =\frac{2\left(i E_{T}-m-m e^{i k_{x}}\right)}{t+\lambda} \psi_{2 N_{y}}, \\
\psi_{2 N_{y}-2-2 j} & =\frac{2\left(i E_{T}-m-m e^{i k_{x}}\right)}{t+\lambda} \psi_{2 N_{y}-2 j}+\frac{t-\lambda}{t+\lambda} \psi_{2 N_{y}+2-2 j}, \\
\psi_{2 N_{y}+1-2 j} & =-i \psi_{2 N_{y}+2-2 j},
\end{aligned}
$$

where $j=1,2,3, \ldots, N_{y}-2$ is the index in the $y$ direction; $\psi_{3}=-i \psi_{4}$, and $\psi_{1}=-i \psi_{2}$.

Figure 4 presents the analytical result and the numerical result of the topological edge states that localize at the left, right, bottom, and top boundaries. The analytical result and the numerical result are well in agreement with each other.
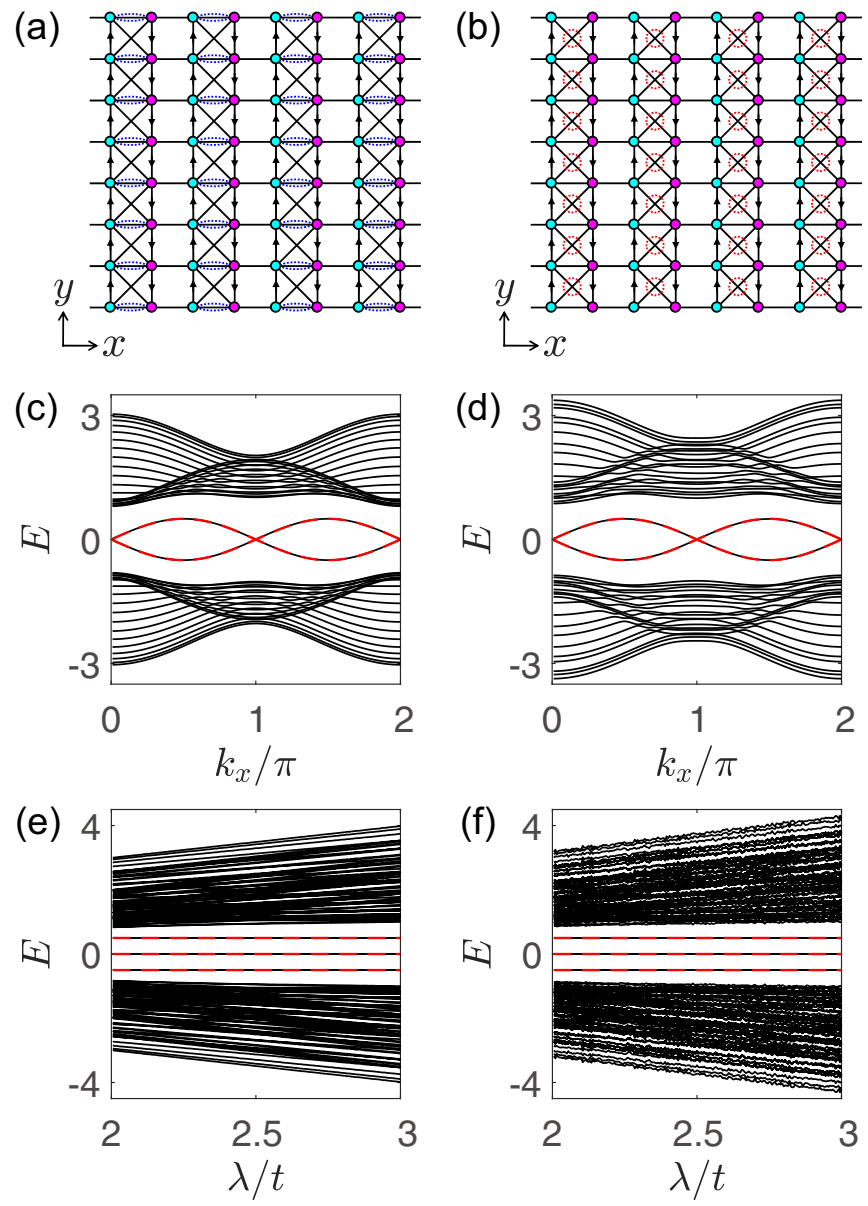

FIG. 5. The disordered 2D square lattice under the PBC in the $x$ direction and the $\mathrm{OBC}$ in the $y$ direction. The perturbations present in the coupling terms. Notably, the disturbed lattice still possesses the particle-hole symmetry but not the inversion symmetry. Without the perturbations, the edge state energies are $\pm m \sin k_{x}$, as marked by the dashed red lines. The perturbations are (a) $m\left(1+0.2 R_{i, j}\right)$ (the dotted blue ellipse) in each unit cell $(i, j) ; R_{i, j}$ is a random real number within the region $[0,1]$. (b) $\lambda\left(1+0.2 R_{i, j}\right)$ (the dotted red circle) for each cross coupling $\lambda$. The perturbations are shown in (a) [(b)] for (c) and (e) [(d) and (f)]. The parameters are $t=1, m=$ $1 / 2$, and $N_{y}=20 ; \lambda=2$ for (c) and (d), and $N_{x}=4$ for (e) and (f).

\section{ROBUSTNESS OF IN-GAP EDGE STATES}

In the anisotropic 2D square lattice, the topological edge states are robust to the system disorders under the particlehole symmetry. In this section, we mainly focus on demonstrating the robustness of the in-gap edge states in the nontrivial phase with a zero Chern number, $C=0$. The in-gap edge states appear under the PBC in the $x$ direction and the OBC in the $y$ direction.

Schematics of the 2D lattice with perturbations are shown in Figs. 5(a) and 5(b). The disorders considered in the real couplings $m$ and $\lambda$ satisfy the particle-hole symmetry. The couplings with perturbations are marked by the blue ellipses and red circles. In Fig. 5(a), the perturbations presented in the intracell coupling (the dotted blue ellipses) are $m(1+$ $\left.0.2 R_{i, j}\right)$, where the subscripts $(i, j)$ are the indices of the unit cell and $R_{i, j}$ are random real numbers within the region [0,1]. 
In Fig. 5(b), the perturbations are chosen as $\lambda\left(1+0.2 R_{i, j}\right)$ for each cross coupling $\lambda$ (the dotted red circles) between the unit cells.

We also perform two types of 2D disorders to unveil the robustness of the in-gap edge states. One type of disorder has the translational symmetry in the $x$ direction; that is, the coupling disorders in different unit cells along the $x$ direction are identical but are different in different unit cells along the $y$ direction. The energy spectra of the 2D lattice for the parameters $t=1, m=1 / 2$, and $\lambda=2$ are depicted in Fig. 5(c) for the disorders presented in the intracell couplings $m$ [Fig. 5(a)] and in Fig. 5(d) for the disorders presented in the intercell coupling $\lambda$ [Fig. 5(b)]. The disorders are chosen to be translationally invariant in the $x$ direction in Figs. 5(c) and 5(d). The system is in the nontrivial phase with a zero Chern number, $C=0$, and the in-gap edge state energies are $\pm m \sin k_{x}$. The edge state energies are unchanged in the presence of coupling perturbations, and the robustness of ingap edge states is verified.

The other type of disorder exhibited does not have the translational symmetry in either the $x$ direction or the $y$ direction. In the absence of perturbations, the momentum is $k_{x}=2 n \pi / N_{x}$, with $n=1,2,3, \ldots, N_{x}$. Taking $N_{x}=4$, the set of discrete $k_{x}$ involves $\pi / 2, \pi, 3 \pi / 2$, and $2 \pi$. The eigenenergies in the absence of perturbations are constituted by all four $k_{x}$ in Fig. 3(d). All the $N=2 N_{x} N_{y}$ eigenenergies of the 2D lattice as functions of the coupling strength $\lambda$ are shown in Figs. 5(e) and 5(f) for the disorders presented in the couplings $m$ [Fig. 5(a)] and the disorders presented in the couplings $\lambda$ [Fig. 5 (b)], respectively, where the parameters are $t=1$ and $m=1 / 2$. The system is in the nontrivial phase with a zero Chern number, $C=0$, and the in-gap edge states with energies $E_{d}= \pm m \sin k_{x}$ exist at $k_{x}=\pi / 2, \pi, 3 \pi / 2$, and $2 \pi$. The dashed red lines are the edge state energies in the systems without perturbations for comparison. Notably, the edge states are robust to the $2 \mathrm{D}$ particle-hole symmetric disorders.

\section{CONCLUSION}

In conclusion, we have investigated an anisotropic 2D lattice that supports topologically nontrivial phases with a zero Chern number. The wave polarization is a topological characterization in the absence of the singularity in the whole $\mathrm{BZ}$ under the properly chosen gauge. The absence and presence of in-gap edge states can be predicted from the zero and half-integer polarizations defined as the integral of the Berry connection in the whole BZ, respectively. The topologically protected in-gap edge states are present inside the band gap and detach the bulk band. The in-gap edge states are robust to the 2D particle-hole symmetric disorders. The in-gap edge states in the nontrivial phase with vanishing Chern numbers do not exist only in the 2D square lattice; further explorations on the 2D honeycomb lattice or multiband systems would also be interesting.

\section{ACKNOWLEDGMENTS}

This work was supported by the National Natural Science Foundation of China (Grants No. 11975128 and No. 11874225).
[1] M. Z. Hasan and C. L. Kane, Colloquium: Topological insulators, Rev. Mod. Phys. 82, 3045 (2010).

[2] X.-L. Qi and S.-C. Zhang, Topological insulators and superconductors, Rev. Mod. Phys. 83, 1057 (2011).

[3] M. Hafezi, E. A. Demler, M. D. Lukin, and J. M. Taylor, Robust optical delay lines with topological protection, Nat. Phys. 7, 907 (2011).

[4] Y. Ran, Y. Zhang, and A. Vishwanath, One-dimensional topologically protected modes in topological insulators with lattice dislocations, Nat. Phys. 5, 298 (2009).

[5] Z. Wang, X.-L. Qi, and S.-C. Zhang, Equivalent topological invariants of topological insulators, New J. Phys. 12, 065007 (2010).

[6] L. J. Lang, X. Cai, and S. Chen, Edge States and Topological Phases in One-Dimensional Optical Superlattices, Phys. Rev. Lett. 108, 220401 (2012); L. J. Lang and S. Chen, Majorana fermions in density-modulated $p$-wave superconducting wires, Phys. Rev. B 86, 205135 (2012).

[7] X.-J. Liu, K. T. Law, T. K. Ng, and P. A. Lee, Detecting Topological Phases in Cold Atoms, Phys. Rev. Lett. 111, 120402 (2013).

[8] D.-L. Deng, S.-T. Wang, C. Shen, and L.-M. Duan, Hopf insulators and their topologically protected surface states, Phys. Rev. B 88, 201105(R) (2013).

[9] N. Goldman, J. Dalibard, A. Dauphin, F. Gerbier, M. Lewenstein, P. Zoller, and I. B. Spielman, Direct imaging of topological edge states in cold-atom systems, Proc. Natl. Acad. Sci. USA 110, 6736 (2013).

[10] M. Atala, M. Aidelsburger, J. T. Barreiro, D. Abanin, T. Kitagawa, E. Demler, and I. Bloch, Direct measurement of the Zak phase in topological Bloch bands, Nat. Phys. 9, 795 (2013).

[11] Y. X. Zhao and Z. D. Wang, Topological connection between the stability of Fermi surfaces and topological insulators and superconductors, Phys. Rev. B 89, 075111 (2014).

[12] D.-L. Deng, S.-T. Wang, and L.-M. Duan, Direct probe of topological order for cold atoms, Phys. Rev. A 90, 041601(R) (2014).

[13] Z. Wang and S.-C. Zhang, Topological Invariants and GroundState Wave Functions of Topological Insulators on a Torus, Phys. Rev. X 4, 011006 (2014).

[14] N. Goldman, J. C. Budich, and P. Zoller, Topological quantum matter with ultracold gases in optical lattices, Nat. Phys. 12, 639 (2016).

[15] C. He, X. Ni, H. Ge, X.-C. Sun, Y.-B. Chen, M.-H. Lu, X.-P. Liu, and Y.-F. Chen, Acoustic topological insulator and robust one-way sound transport, Nat. Phys. 12, 1124 (2016).

[16] Z. Yan, R. Bi, and Z. Wang, Majorana Zero Modes Protected by a Hopf Invariant in Topologically Trivial Superconductors, Phys. Rev. Lett. 118, 147003 (2017).

[17] Y.-B. Yang, L.-M. Duan, and Y. Xu, Continuously tunable topological pump in high-dimensional cold atomic gases, Phys. Rev. B 98, 165128 (2018). 
[18] S.-Y. Yu, C. He, Z. Wang, F.-K. Liu, X.-C. Sun, Z. Li, H.-Z. Lu, M.-H. Lu, X.-P. Liu, and Y.-F. Chen, Elastic pseudospin transport for integratable topological phononic circuits, Nat. Commun. 9, 3072 (2018).

[19] W. Sun, C.-R. Yi, B.-Z. Wang, W.-W. Zhang, B. C. Sanders, X.-T. Xu, Z.-Y. Wang, J. Schmiedmayer, Y. Deng, X.-J. Liu, S. Chen, and J.-W. Pan, Uncover Topology by Quantum Quench Dynamics, Phys. Rev. Lett. 121, 250403 (2018).

[20] X. Ni, M. Weiner, A. Alù, and A. B. Khanikaev, Observation of higher-order topological acoustic states protected by generalized chiral symmetry, Nat. Mater. 18, 113 (2019).

[21] X.-D. Chen, W.-M. Deng, F.-L. Shi, F.-L. Zhao, M. Chen, and J.-W. Dong, Direct Observation of Corner States in SecondOrder Topological Photonic Crystal Slabs, Phys. Rev. Lett. 122, 233902 (2019).

[22] B.-Y. Xie, G.-X. Su, H.-F. Wang, H. Su, X.-P. Shen, P. Zhan, M.-H. Lu, Z.-L. Wang, and Y.-F. Chen, Visualization of HigherOrder Topological Insulating Phases in Two-Dimensional Dielectric Photonic Crystals, Phys. Rev. Lett. 122, 233903 (2019).

[23] B.-Y. Xie, H.-F. Wang, X.-Y. Zhu, M.-H. Lu, Z. D. Wang, and Y.-F. Chen, Photonics meets topology, Opt. Express 26, 24531 (2018).

[24] D.-W. Zhang, Y.-Q. Zhu, Y. X. Zhao, H. Yan, and S.-L. Zhu, Topological quantum matter with cold atoms, Adv. Phys. 67, 253 (2019).

[25] N. R. Cooper, J. Dalibard, and I. B. Spielman, Topological bands for ultracold atoms, Rev. Mod. Phys. 91, 015005 (2019).

[26] T. Ozawa, H. M. Price, A. Amo, N. Goldman, M. Hafezi, L. Lu, M. C. Rechtsman, D. Schuster, J. Simon, O. Zilberberg, and I. Carusotto, Topological photonics, Rev. Mod. Phys. 91, 015006 (2019).

[27] Y. Xu, Topological gapless matters in three-dimensional ultracold atomic gases, Front. Phys. 14, 43402 (2019).

[28] S. Mittal, S. Ganeshan, J. Fan, A. Vaezi, and M. Hafezi, Measurement of topological invariants in a 2D photonic system, Nat. Photonics 10, 180 (2016).

[29] X.-J. Liu, K. T. Law, and T. K. Ng, Realization of 2D Spin-Orbit Interaction and Exotic Topological Orders in Cold Atoms, Phys. Rev. Lett. 112, 086401 (2014).

[30] Y.-J. Wu, J. He, C.-L. Zang, and S.-P. Kou, Topological superfluid state of fermions on a $p$-band optical square lattice, Phys. Rev. B 86, 085128 (2012).

[31] P. Wang, Z.-Q. Yu, Z. Fu, J. Miao, L. Huang, S. Chai, H. Zhai, and J. Zhang, Spin-Orbit Coupled Degenerate Fermi Gases, Phys. Rev. Lett. 109, 095301 (2012).

[32] H. Zhai, Spin-orbit coupled quantum gases, Int. J. Mod. Phys. B 26, 1230001 (2012).

[33] J. He, Y.-X. Zhu, Y.-J. Wu, L.-F. Liu, Y. Liang, and S.-P. Kou, Zero modes around vacancies in topological insulators and topological superconductors on the honeycomb lattice with particle-hole symmetry, Phys. Rev. B 87, 075126 (2013).

[34] W.-J. Chen, S.-J. Jiang, X.-D. Chen, B. Zhu, L. Zhou, J.-W. Dong, and C. T. Chan, Experimental realization of photonic topological insulator in a uniaxial metacrystal waveguide, Nat. Commun. 5, 5782 (2014).

[35] Y.-J. Wu, J. He, and S.-P. Kou, Topological mid-gap states of Chern insulator with flux-superlattice, Europhys. Lett. 105, 47002 (2014).

[36] C. He, X.-C. Sun, X.-P. Liu, M.-H. Lu, Y. Chen, L. Feng, and Y.-F. Chen, Photonic topological insulator with broken time-reversal symmetry, Proc. Natl. Acad. Sci. USA 113, 4924 (2016).

[37] B. Yang, T. Wu, and X. Zhang, Topological properties of nearly flat bands in two-dimensional photonic crystals, J. Opt. Soc. Am. B 34, 831 (2017).

[38] B. Yang, T. Wu, and X. Zhang, Engineering topological edge states in two dimensional magnetic photonic crystal, Appl. Phys. Lett. 110, 021109 (2017).

[39] B. Wang, T. Chen, and X. Zhang, Experimental Observation of Topologically Protected Bound States with Vanishing Chern Numbers in a Two-Dimensional Quantum Walk, Phys. Rev. Lett. 121, 100501 (2018).

[40] K. V. Klitzing, G. Dorda, and M. Pepper, New Method for High-Accuracy Determination of the Fine-Structure Constant Based on Quantized Hall Resistance, Phys. Rev. Lett. 45, 494 (1980).

[41] D. J. Thouless, M. Kohmoto, M. P. Nightingale, and M. den Nijs, Quantized Hall Conductance in a Two-Dimensional Periodic Potential, Phys. Rev. Lett. 49, 405 (1982).

[42] F. D. M. Haldane, Model for a Quantum Hall Effect without Landau Levels: Condensed-Matter Realization of the "Parity Anomaly,'Phys. Rev. Lett. 61, 2015 (1988).

[43] S. M. Girvin, Particle-hole symmetry in the anomalous quantum Hall effect, Phys. Rev. B 29, 6012 (1984).

[44] W. P. Su, Ground-state degeneracy and fractionally charged excitations in the anomalous quantum Hall effect, Phys. Rev. B 30, 1069 (1984).

[45] H. B. Laughlin, Anomalous Quantum Hall Effect: An Incompressible Quantum Fluid with Fractionally Charged Excitations, Phys. Rev. Lett. 50, 1395 (1983).

[46] B. J. van Wees, E. M. M. Willems, C. J. P. M. Harmans, C. W. J. Beenakker, H. van Houten, J. G. Williamson, C. T. Foxon, and J. J. Harris, Anomalous Integer Quantum Hall Effect in the Ballistic Regime with Quantum Point Contacts, Phys. Rev. Lett. 62, 1181 (1988).

[47] H. Weng, R. Yu, X. Hu, X. Dai, and Z. Fang, Quantum anomalous Hall effect and related topological electronic states, Adv. Phys. 64, 227 (2015).

[48] M. Ezawa, Valley-Polarized Metals and Quantum Anomalous Hall Effect in Silicene, Phys. Rev. Lett. 109, 055502 (2012); Spin valleytronics in silicene: Quantum spin Hall-quantum anomalous Hall insulators and single-valley semimetals, Phys. Rev. B 87, 155415 (2013).

[49] X. L. Qi, Y. S. Wu, and S. C. Zhang, Topological quantization of the spin Hall effect in two-dimensional paramagnetic semiconductors, Phys. Rev. B 74, 085308 (2006).

[50] A. B. Khanikaev and G. Shvets, Two-dimensional topological photonics, Nat. Photonics 11, 763 (2017).

[51] B. A. Bernevig and T. L. Hughes, Topological Insulators and Topological Superconductors (Princeton University Press, Princeton, NJ, 2013).

[52] J. K. Asbóth, L. Oroszlány, and A. Pályi, A Short Course on Topological Insulators: Band Structure and Edge States in One and Two Dimensions, Lecture Notes in Physics Vol. 919 (Springer, Cham, 2016).

[53] L. Lu, J. D. Joannopoulos, and M. Soljačić, Topological photonics, Nat. Photonics 8, 821 (2014).

[54] F. Liu and K. Wakabayashi, Novel Topological Phase with a Zero Berry Curvature, Phys. Rev. Lett. 118, 076803 (2017). 
[55] R. Resta, Macroscopic polarization in crystalline dielectrics: The geometric phase approach, Rev. Mod. Phys. 66, 899 (1994).

[56] A. Altland and M. R. Zirnbauer, Nonstandard symmetry classes in mesoscopic normal-superconducting hybrid structures, Phys. Rev. B 55, 1142 (1997).

[57] A. P. Schnyder, S. Ryu, A. Furusaki, and A. W. W. Ludwig, Classification of topological insulators and superconductors in three spatial dimensions, Phys. Rev. B 78, 195125 (2008).
[58] S. Ryu, A. P. Schnyder, A. Furusaki, and A. W. W. Ludwig, Topological insulators and superconductors: Tenfold way and dimensional hierarchy, New J. Phys. 12, 065010 (2010).

[59] M. Creutz, End States, Ladder Compounds, and Domain-Wall Fermions, Phys. Rev. Lett. 83, 2636 (1999).

[60] D. Xiao, M.-C. Chang, and Q. Niu, Berry phase effects on electronic properties, Rev. Mod. Phys. 82, 1959 (2010).

[61] J. Zak, Berry's Phase for Energy Bands in Solids, Phys. Rev. Lett. 62, 2747 (1989). 